Case Report

\title{
Auscultating a pea: the stethoscope may matter in otorhinolaryngology emergency evaluation
}

\author{
Francisco Alves De Sousa*, Ana Costa Silva, Ana Nóbrega Pinto, Cecília Almeida E. Sousa
} Department of Otorhinolaryngology and Head and Neck surgery, Centro Hospitalar Universitário do Porto, Porto,
Portugal

Received: 30 December 2020

Revised: 01 February 2021

Accepted: 09 February 2021

*Correspondence:

Dr. Francisco Alves de Sousa,

E-mail: franciscoalvesousa@gmail.com

Copyright: ( the author(s), publisher and licensee Medip Academy. This is an open-access article distributed under the terms of the Creative Commons Attribution Non-Commercial License, which permits unrestricted non-commercial use, distribution, and reproduction in any medium, provided the original work is properly cited.

\begin{abstract}
Foreign body sensation is a common complaint in the otorhinolaryngology emergency. Careful examination of the patient's pharynx is mandatory, but sometimes the object is not visualized. In such scenario, it may be important to explore signs and symptoms indicating lower aerodigestive impaction. This work describes the case of a 73-year-old woman without relevant comorbidities attending to emergency care. She complained of a foreign body sensation on the right side of the throat after ingesting a meal, which motivated referral to otorhinolaryngology. Flexible transnasal nasopharyngoscopy was unremarkable and no foreign bodies were found. Auscultation was performed revealing lowpitch expiratory wheezing on her right hemithorax. The suspicion of bronchial foreign body was then raised, which was ultimately confirmed by imaging and bronchoscopy, showing an impacted pea on the right lower lobe bronchus. The stethoscope was hence determinant for detecting aspiration, by revealing consistent alterations. Its usage should be encouraged in similar scenarios, highlighting the role of this classic but sometimes forgotten tool. Importantly, higher neck/throat sensations should not exclude the possibility of a lower airway foreign body.
\end{abstract}

Keywords: Stethoscope, Foreign body, Aspiration, Throat sensation

\section{INTRODUCTION}

Foreign body sensation is a common complaint in the otorhinolaryngology emergency, so that the otolaryngologist is the frontline assistant doctor in these situations. Careful examination of the patient's pharynx is mandatory, but sometimes the problem lies deep further in the digestive or respiratory tract. Not rarely, the throughout examination does not reveal the object. ${ }^{1}$ The workup of this situations may be a challenge due to the vast clinical scenarios implied: from the sole complaint of a psychogenic conversion to an actual impacted foreign body in the aerodigestive tract. ${ }^{1,2}$ An holistic approach with a careful examination may be the clue for managing this conditions, in order to assure optimal diagnosis and treatment while avoiding serious complications. ${ }^{2}$ Two hundred years ago Laennec, a young French physician, discovered the stethoscope, in order to allow lung auscultation. The stethoscope is an emblematic appendix of physicians ever since. ${ }^{3}$ But can it be useful in a case of foreign body sensation in the throat?

\section{CASE REPORT}

We report the case of a 73-year-old-woman with no relevant comorbidities presenting to the Otorhinolaryngology emergency department with a foreign body sensation on the right side of the throat. Two hours before she had suffered from an episode of choking during lunch. The episode resolved, but a foreign body sensation on her right submandibular region persisted, impelling the search for medical assistance. The oropharynx examination, indirect laryngoscopy and transnasal flexible fiber optic nasopharyngoscopy were 
unremarkable, and no pharyngeal stasis was present. The patient denied dyspnea and was visibly comfortable speaking. Nevertheless, occasional bouts of coughing were noted during her observation. Auscultation was hence performed: revealing diffuse low-pitched expiratory wheezing on the right hemithorax. Her oxygen saturation was then measured showing a $93 \%$ value. A high suspicion of bronchial aspiration was raised after the auscultatory findings and a thoracic CT scan was performed (Figure 1) confirming an impacted foreign body in the right bronchial tree; after bridging with the interventional Pneumology team, a Bronchoscopy was performed (Figure 2), with successful removal of a pea using biopsy forceps. The patient was discharged with antibiotic and prednisolone regimen on the next day without complaints. One month after first observation the patient was clinically stable.

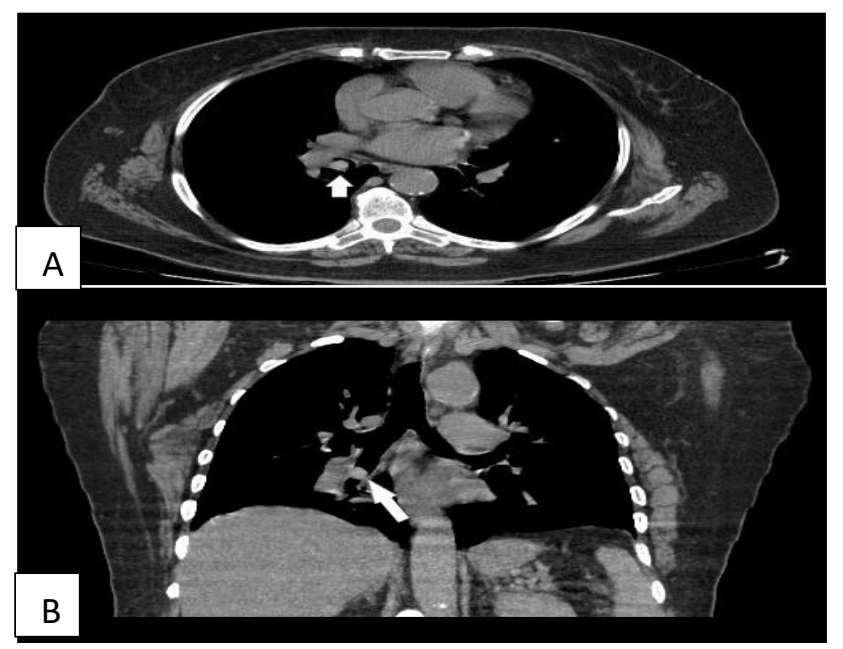

Figure 1: (A) Axial thoracic CT scan of a rounded foreign body in the right lower lobe bronchus (arrow).

(B) Coronal thoracic CT scan of a $10 \mathrm{~mm}$ rounded foreign body in the right lower lobe bronchus (arrow).

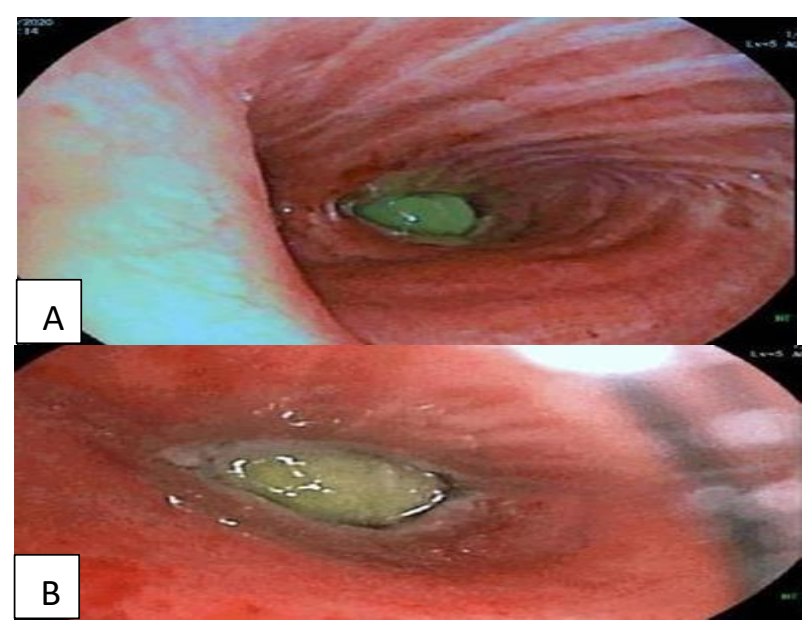

Figure 1: (A) Bronchoscopy of a pea occluding right lower intermediate bronchus; (B) bronchoscopy close up revealing pea involved by mucous secretions. After removal the mucosa showed residual inflammatory signs without basal membrane affection.

\section{DISCUSSION}

Studies suggest that when a patient is able to lateralize a presumptive foreign body within the cervical region the object is likely to be found above the cricopharyngeal "muscle" and on the side indicated. ${ }^{4}$ It is known that impacted foreign bodies in the esophagus may be referred as a neck/throat sensation, and a similar complaint may also be produced by a pneumothorax or pneumomediastinum. ${ }^{1,4}$ Curiously, the impression reported by the patient was on the same side of the impacted foreign body in the lower respiratory tract. We have not found any similar reports regarding lower airway obstruction and throat sensations. ${ }^{5,6}$ Airway protection relies upon a variety of highly coordinated neural reflex responses that ultimately help to remove or expel the harmful stimuli. ${ }^{6}$ The activation of bronchopulmonary C-fibers occurs in mechanical distention of the airways. ${ }^{7}$ One study reports that stimulation of this afferent fibers may also elicit burning and choking sensation in the throat, neck and upper chest, which could help to explain the clinical presentation on this case. ${ }^{5}$ It is important to highlight that neck discomfort should not exclude the suspicion of a lower airway obstruction, when other (even mild) clinical signs are present.

Aspiration of foreign bodies can occur both in children and adults. Children are prone to aspiration due to their inherent curiosity impelling them to put objects on the mouth. There are different risk factors for aspiration in adults, such as alcohol consumption, drug consumption, senility, seizure, trauma, cognitive impairment, dysphagia syndromes, or artificial dentures. In some rarer cases, such as the one here described, adulthood aspiration may happen accidentally in individuals without known risk factors. ${ }^{8}$ Foreign-body aspiration in adults can be difficult to diagnose because the symptoms are nonspecific and chest X-rays may be normal due to organic composition of the foreign bodies. ${ }^{9}$ Thus, it is fundamental for clinicians dealing with these exceptional scenarios to be aware of this possibility and use all the reachable tools to make a proper diagnosis. "Stethoscope auscultation may be determinant in this context, and should not be forgotten". "A proper diagnosis" will allow further articulation with the pneumology team for the best final treatment solution. If left untreated, patients may face a real risk of atelectasis, post obstructive pneumonia or lung abscess. ${ }^{8}$

\section{CONCLUSION}

The reference to a foreign body sensation in the neck acted as a potential confounder in the clinical presentation of this case, so as the relative paucity of symptoms. The use of stethoscope was determinant for raising the suspicion of aspiration by revealing consistent alterations. Additionally, it also provided an anatomical clue for lateralization to the radiologist. Hence, its usage should be encouraged in similar scenarios. Importantly, 
higher neck/throat sensations should not exclude the possibility of a lower airway foreign body.

Funding: No funding sources

Conflict of interest: None declared

Ethical approval: Not required

\section{REFERENCES}

1. Mathew P, Tiwari R, David J, Tiwari H. MEDICAL Short Review A Review on Foreign Body Obstruction in Throat and A Case of Molar Tooth in Esophagus. 2016;3(3):469-71.

2. Arabi Mianroodi A, Teimouri Y, Vallance NA. Foreign bodies: aspirated or ingested? A report of two unusual cases. Iran $\mathbf{J}$ Otorhinolaryngol. 2012;24(67):91-4.

3. David L, Dumitrascu DL. The bicentennial of the stethoscope: a reappraisal. Clujul Med. 2017;90(3):361-3.

4. Connolly AA, Birchall M, Walsh-Waring GP, Moore-Gillon V. Ingested foreign bodies: patientguided localization is a useful clinical tool. Clin Otolaryngol Allied Sci. 1992;17(6):520-4.
5. Lee L-Y. Respiratory sensations evoked by activation of bronchopulmonary C-fibers. Respir Physiol Neurobiol. 2009;167(1):26-35.

6. Mazzone SB. An overview of the sensory receptors regulating cough. Cough. 2005;1:2.

7. Undem BJ, Kollarik M. The role of vagal afferent nerves in chronic obstructive pulmonary disease. Proc Am Thorac Soc. 2005;2(4):355-72.

8. Afghani R. Unusual aspiration of foreign body in adults. Ann Cardiovasc Thorac Surg. 2018;01(01):810 .

9. Bain A, Barthos A, Hoffstein V, Batt J. Foreignbody aspiration in the adult: presentation and management. Can Respir J. 2013;20(6):e98-9.

Cite this article as: De Sousa FA, Silva AC, Pinto AN, E Sousa CA. Auscultating a pea: the stethoscope may matter in otorhinolaryngology emergency evaluation. Int J Otorhinolaryngol Head Neck Surg 2021;7:530-2. 